\title{
Research on the Comment Psychology of Music Applications Under the Uses and Gratification Approach
}

\author{
Yuting $\mathrm{Li}^{1{ }^{1, *}}$ Minling Yang ${ }^{2}$ \\ ${ }^{1}$ School of Art and Design, Guangdong University of Finance \& Economics, Guangzhou, Guangdong, China \\ ${ }^{2}$ School of Media and Communication, Shenzhen University, Shenzhen, Guangdong, China \\ "Corresponding author. Email: Yuting_Li@gdufe.edu.cn
}

\begin{abstract}
The study adopts quantitative and qualitative comprehensive research methods, captures users' music comments through the octopus collector, analyzes the emotional tendency of the music application (app) comment area and the three core psychological motivations of the user's using behavior of music application comment based on the Uses and Gratification Approach, and on this basis, constructs a psychological motivation model of music application users' using behaviors. Studies have found that social needs are the most important psychological motivation for music application users to participate in comments, emotional needs are the key psychological motivation, and cognitive needs play an important role in driving users' behavior in participating in comments.
\end{abstract}

Keywords: Music applications, Music comments, Users, Psychological motivations, Text analysis.

\section{INTRODUCTION}

The number of people who have access to and use the Internet in the world has reached 4.5 billion, and the number of social media users has exceeded 3.8 billion, symbolizing that social media has gradually been embedded in everyone's daily life. However, with the development of social media platforms becoming more and more mature, people have begun to get tired of pure social platforms. Vertical social media platforms have gained new development space. Music apps open the "music social contact" mode, that is, "social contact" is no longer independent of music, but embedded in it, and using music as a medium, it stimulates users to enjoy music and engage in social activities.

E. Katz pointed out that the audience contacts and uses the media based on personal needs and desires. The influence of the media on the audience is only in providing information, but the media has no say in whether the audience uses the media or

*Fund: This article is the result of the 2020 Guangdong Province Academic Degrees and Postgraduate Education Reform Research Project "Research on the Construction Management System and Mechanism of the Joint Training Postgraduate Demonstration Base Based on Process Control" (2020JGXM052). what information they choose. At the same time, in the age of social media, technological empowerment has liquefied the boundaries of communication. "Audience" should become a "sender" or "user", which has more initiative and individual characteristics than the audience. Therefore, the user's psychology is complicated.

At present, the research on the psychology of social media users has attracted much attention in the academic field and has produced rich results. The user psychology of music social media is also an indispensable part of social media user psychology research. This article will take the NetEase Cloud Music application as an example, and take the music comments of ordinary users in NetEase Cloud Music as the research object. Based on the Uses and Gratification Approach, this article explores the following questions: Are the emotional tendencies of NetEase Cloud Music's commentary area positive or negative? What kind of psychology and needs do NetEase Cloud Music users participate in comments based on? As one of the most important music social media platforms in China, the research on Netease Cloud Music's user psychology has an important reference for the 
development of the "music + social contact" model and other music social media platforms.

\section{RESEARCH PATH AND DATA ANALYSIS}

\subsection{Design and Sampling}

The research object of this article is user comments of songs on the music application

Table 1. Top ten songs on the official hot song list of the music application

\begin{tabular}{llll}
\hline Ranking & \multicolumn{1}{c}{ Song title and singer } & Amount of comments & $\begin{array}{c}\text { Selected amount of } \\
\text { comments }\end{array}$ \\
\hline 1 & For ya (Jiang Xiaoni) & 40,994 & 1,000 \\
2 & Step Across Mountains and Rivers (It's Uncle 7) & 89,453 & 1,000 \\
3 & He Just Went By (h3R3/Gao Xu) & 142,709 & 1,000 \\
4 & Shutdown and Shutdown (Yang Yang) & 63,644 & 1,000 \\
5 & Your Silhouette (Ye Qionglin) & 28,146 & 1,000 \\
6 & Xi (Ge Dongqi) (Santa_SA/Ma Ye) & 202,269 & 1,000 \\
7 & Fox's Fairy Tale (Sant Sa & 1,000 \\
8 & Night Breeze (Copy/BT07-PZ) & 40,950 & 1,000 \\
9 & Mang (Li Runqi) & 114,985 & 1,000 \\
10 & Missed Lover (Accusefive) & 123,486 & 1,000 \\
\hline
\end{tabular}

\subsection{Screening Sample}

After sorting the 10,000 comments of each song in descending order according to the heat (ie the number of likes), reading the comment text one by one, excluding empty text samples and non-Chinese samples, and selecting the top 1,000 comments for each song according to the heat, finally, a total of 10,000 valid comments are obtained.

\subsection{Word Frequency Analysis}

The word frequency statistics of music application users' comment text can directly reflect the emotional tendency of music application users to songs and platform comment area. After importing 10,000 user comment texts into the Nvivo11 software, using the search function of the software, setting the word frequency condition as synonym matching, and removing meaningless words (setting stop words), the full text word frequency set and high-frequency word cloud figure can be got, as shown in "Table 2". The analysis results show that, overall, in the user review text of the music application comment area, positive words, such as "like", "come on", "happy", etc. dominate. platform, selecting the user comments of the top ten songs on the official hot song list in the music application ranking for sampling (the data of the hot song list is as of February 11, 2021). Since the number of comments captured by the octopus collector software for each song is limited to less than 10,000 , this study obtained a total of 100,000 song comment texts based on ten songs. The sample selection is shown in "Table 1". 


\begin{tabular}{|c|c|c|}
\hline Negative emotion & 2041 & $17.93 \%$ \\
\hline \multicolumn{3}{|c|}{ Among them, the segmented statistical results of positive emotion are as follows: } \\
\hline Generally $(0-10)$ & 3086 & $27.11 \%$ \\
\hline Moderately (10-20) & 1391 & $12.22 \%$ \\
\hline Highly (above 20) & 1145 & $10.06 \%$ \\
\hline \multicolumn{3}{|c|}{ Among them, the segmented statistical results of negative emotion are as follows: } \\
\hline Generally $(-10-0)$ & 1291 & $11.34 \%$ \\
\hline Moderately (-20--10) & 465 & $4.09 \%$ \\
\hline Highly (Below -20) & 108 & $0.95 \%$ \\
\hline
\end{tabular}

\subsubsection{Text Encoding and Analysis}

In this study, 10,000 song comments in the music application are imported into Nvivo11, and user comment text is coded to explore the psychological motivations of music application users' behaviors. After preliminary coding, it forms concepts frequently mentioned in 36 music application user comments, such as sharing stories, declaring emotional states, expressing blessings or encouragement, loving songs, sharing knowledge, etc., which are marked as free nodes. Then, it searches for the association between the concepts in 36 free nodes, and classifies them into the same category, forming 9 main concepts and marking them as child nodes. Finally, it further summarizes the 9 main concepts into more systematic core concepts, and finally forms social needs, emotional needs, and cognitive needs, and marks them as tree nodes. The coded data is summarized as shown in "Table 3". The coding results are shown in "Figure $1 "$.

Table 3. Summary of data coding

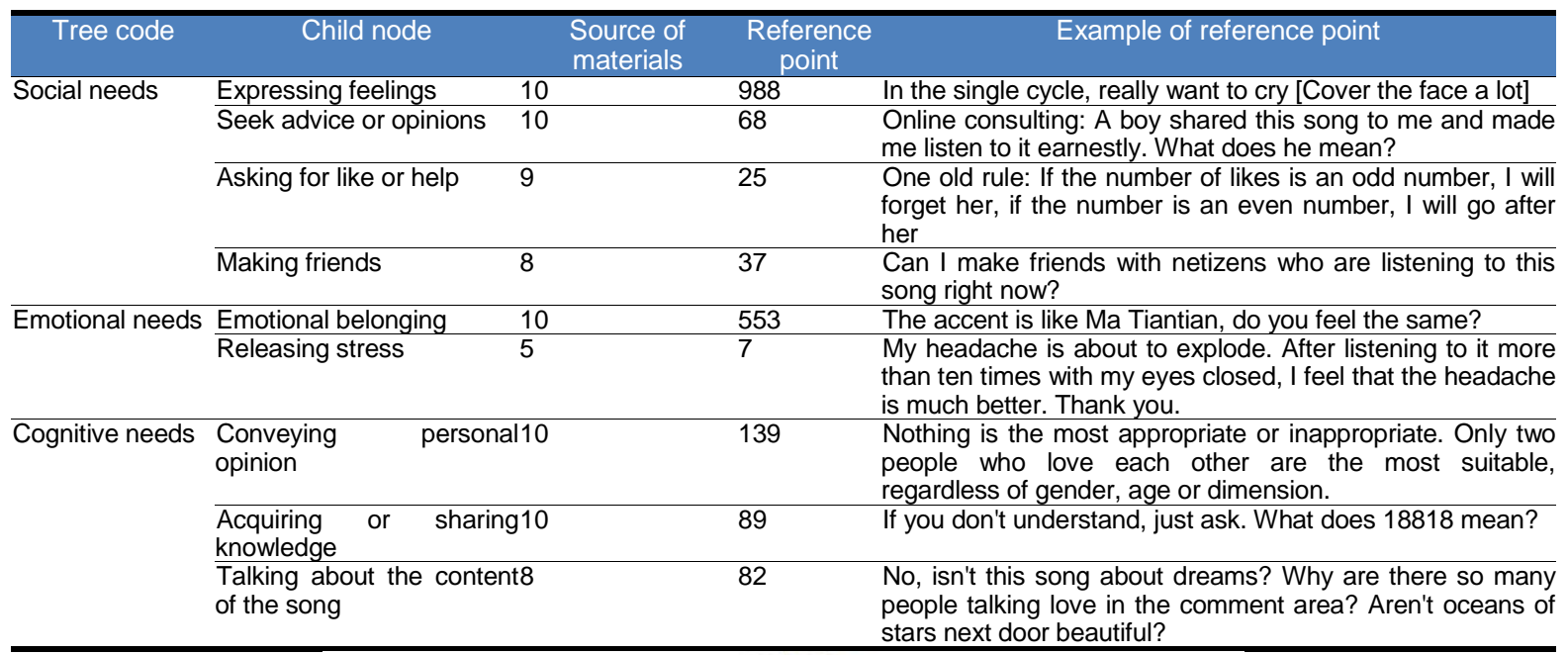

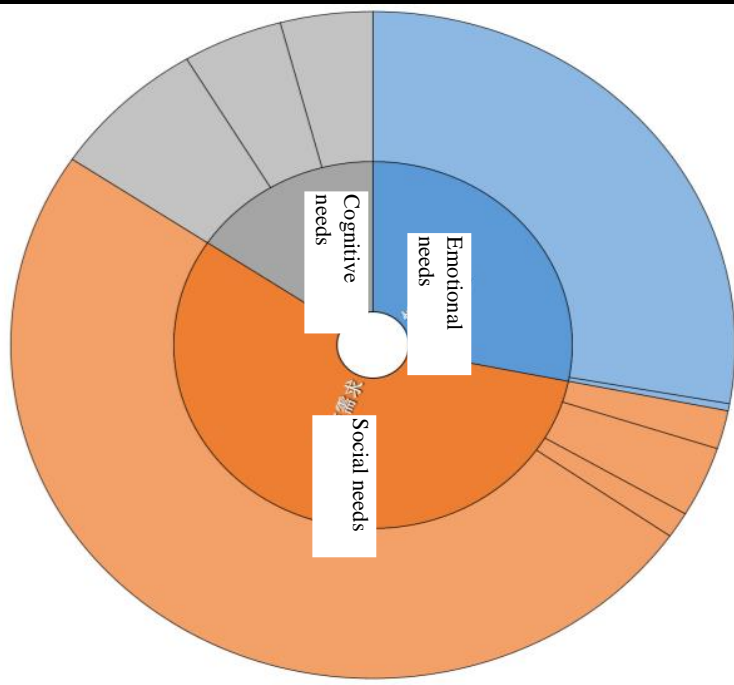

Figure 1 Visualized hierarchical chart of music comment text encoding results. 


\subsubsection{Theoretical Model Construction}

After text coding, the relationship structure between the core concept and the main concept and the concept is basically clear. It studies the psychological motivation model of users' use behavior in the comment area of music application based on the concept-based relational structure constructivism, see "Figure 2".

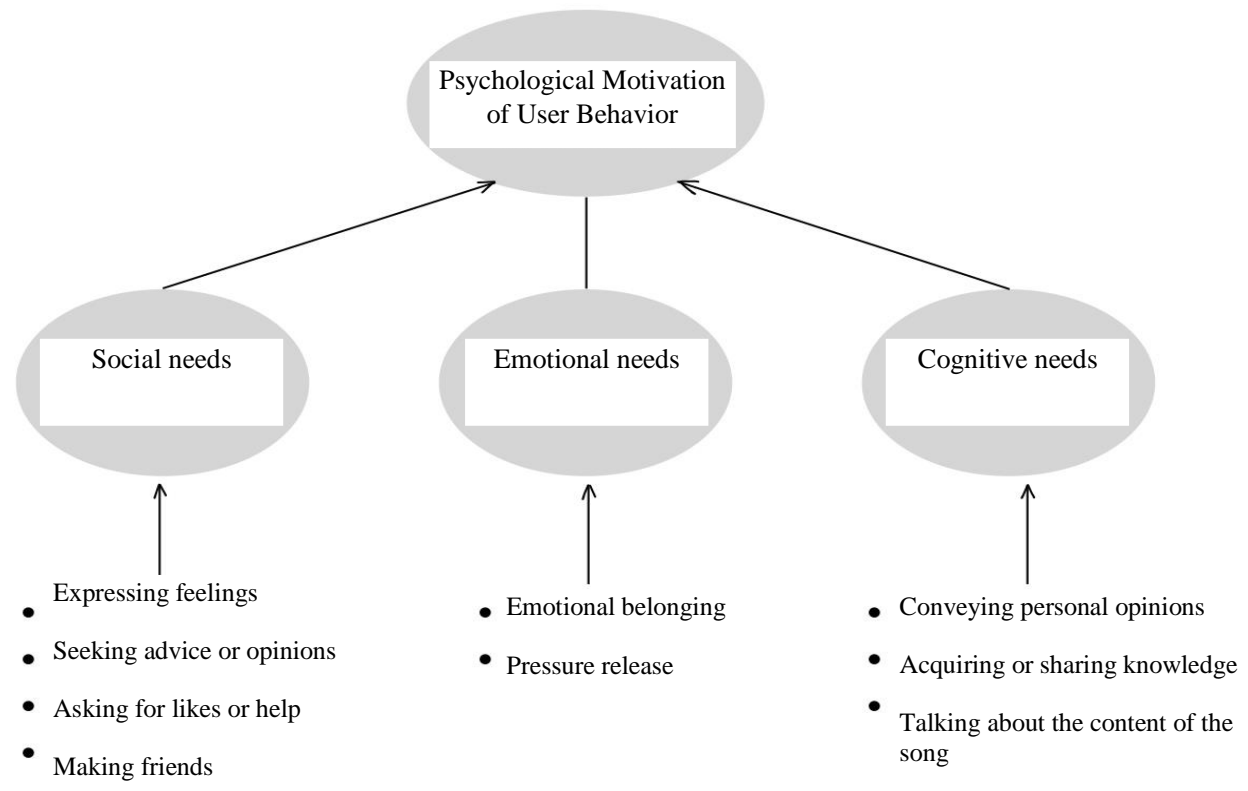

Figure 2 Psychological motivation model of users' behavior in the comment area of the music application.

\section{RESEARCH FINDINGS}

\subsection{Deconstruction and Reconstruction of the Music Comment Area of the Music Application}

Since the establishment of the music application platform, it has become a "public space" for users to express their emotions with its music social model of "listening to music and watching comments". However, with its continuous development, the negative emotions in UGC (user generated content) have become more prominent, and the content of negative emotions has been inundant. Netizens joke that the comment area has become a "pain literature area". Excessive "mourning culture" sharing and similar or even copied tragic stories greatly consume the sympathy and attention of ordinary users of music applications, weakening the value of the music application comment area as a "public space" for emotional expression.
Under the "treatment" of the Cloud Village Healing Project, the UGC in the comment area of the music application generally presents a positive and active atmosphere. Through the use of NVivo11 and ROSTCM6 software to carry out word frequency statistics and sentiment analysis on 10,000 music comment texts, it is found that overall, positive vocabulary and positive emotions dominate. Therefore, in general, many comments express the love of songs, encouragement and best wishes to others, and sharing of their own opinions. The overall atmosphere of the user comment area is positive and active.

\subsection{Analysis of Psychological Motivation of Users in the Comment Area of the Music Application}

\subsubsection{Social Needs}

Human beings are the sum of all social relations and are in connection with others all the time. Everyone has social needs. The comment area of music applications uses music as the carrier, and 
connects the resonance and emotion generated by music to gather users and interact through functions such as comments, likes, appreciation, and hugs, so as to meet people's social needs. Therefore, social needs are the most important psychological motivation for users in the comment area of music applications, including expressing feelings, seeking advice or opinions, asking for likes or help, and making friends. It can be seen from the coding results that the social demand coding reference points account for the highest proportion of $56.2 \%$.

\subsubsection{Emotional Needs}

People have the need to express their emotions. Music itself has the function of meeting personal emotional needs. The emotional core endowed by music itself often inspires the user's true feelings at a certain moment. The music comment area of the music application under the "music + social contact" mode further provides a platform for expressing personal emotions and resonating with others, so as to be comforted and satisfy emotional needs. Therefore, satisfying personal emotional needs is also one of the important psychological motivations for users in the comment area of music applications, including emotional belonging and pressure release. From the coding results, it can be found that the proportion of reference points for emotional need coding is $28.2 \%$, occupying the second place among the three core psychological motivations.

In terms of emotional belonging, the main psychological motivations for users to participate in comments include three: sharing stories, declaring emotional states, and making wishes. Users expect to gain a sense of identity, resonance and companionship from other users by sharing personal stories, emotions and expectations. In terms of stress release, sharing and commenting to relieve pressure in real life is one of the psychological reasons for users to participate in comments. Although the intensity of the purpose of stress release expressed in user comments is not high, the number of users who actually achieve stress release from commenting or viewing comments is not small. Due to the limitation of research methods, there is a large deviation in the study of the intensity of the psychological motivation.

\subsubsection{Cognitive Needs}

People have a tendency to expect more certainty about the unknown. The music comment area of the music application forms a community with music as the focus. The community can achieve efficient information exchange and new knowledge acquisition by posting comments and commenting on others, so as to meet the cognitive needs of users. Cognitive needs are one of the important psychological motivations of users' behavior in the comment area of music applications, including conveying personal opinions, acquiring or sharing knowledge, and talking about the content of the song. It can be known from the coding results that the cognitive need coding reference points account for the least, which is $15.6 \%$. Song themes or lyrics often trigger users to participate in comments, express opinions or raise questions.

\section{CONCLUSION}

This research shows through quantitative and qualitative comprehensive research that nowadays, the comment area of music applications shows a positive and active atmosphere as a whole, and the negative effects brought about by the phenomenon of "Net Depress Cloud" are weakening; at the same time, it is learned through analysis that the psychological motivations of users in the music application comment area include social needs, emotional needs, and cognitive needs. E. Katz emphasized that people contact the media based on specific needs and motives. People all have social, emotional and cognitive needs. Music applications exactly perceive users' needs and set up music products and functions based on these specific needs that can satisfy users' needs. Need gratification encourages users to form a positive media impression of music applications, which in turn increases the frequency of media contact under positive media expectations. Therefore, the reasons for the rise of music social media are not hard to imagine.

\section{AUTHORS' CONTRIBUTIONS}

Yuting $\mathrm{Li}$ contributed to topic design and revising and editing, Minling Yang is responsible for research design and wrote the manuscript. 


\section{REFERENCES}

[1] Hu Zhenzhen. Literature Review of Uses and Gratification Approach [J]. Journal of News Research, 2017,8(08):107. (in Chinese)

[2] Pan Ji. Restore the Active Relationship Between Human Beings and Technology: The Reflection on The Theory of Use and Gratification[J].Journal of International Communication. 2016,38(09):75-85.

[3] Sebastián Valenzuela, Martina Piña, Josefina Ramírez. Behavioral Effects of Framing on Social Media Users: How Conflict, Economic, Human Interest, and Morality Frames Drive News Sharing. 2017, 67(5):803-826.

[4] Peng Lan. Connection and Anti-connection: The Swing of Internet Rules [J]. Journal of International Communication, 2019, 41(02): 20-37. (in Chinese)

[5] Bao Dai, Ahsan Ali, Hongwei Wang. Exploring information avoidance intention of social media users: a cognition-affectconation perspective. 2020, 30(5):1455-1478.

[6] You Yongheng. The Social and Psychological thinking on "Notease cloud music"[N]. Journal of Global Times. 2020-07-31(015). 